"This document is the Accepted Manuscript version of a Published Work that appeared in final form in NANO LETTERS, copyright (C) American Chemical Society after peer review and technical editing by the publisher. To access the final edited and published work see:

https://doi.org/10.1021/acs.nanolett.5b04280" 


\title{
On-surface engineering of a magnetic organometallic nanowire
}

\author{
Maider Ormaza, ${ }^{* \dagger}$ Roberto Robles, ${ }^{\ddagger}$ Nicolas Bachellier, ${ }^{\dagger}$ Paula Abufager,,$+\uparrow$ \\ Nicolás Lorente, ${ }^{\ddagger}, \S, \|$ and Laurent Limot $^{\dagger}$
}

IPCMS, CNRS UMR 7504, Université de Strasbourg, 67034 Strasbourg, France, ICN2 Institut Catala de Nanociencia i Nanotecnologia, Campus UAB, 08193 Bellaterra (Barcelona), Spain, Instituto de Física de Rosario, Consejo Nacional de Investigaciones Científicas y Técnicas (CONICET) and Universidad Nacional de Rosario, Av. Pellegrini 250 (2000) Rosario, Argentina, Centro de Física de Materiales CFM/MPC (CSIC-UPV/EHU), Paseo Manuel de Lardizabal 5, 20018 Donostia-San Sebastián, Spain, and Donostia International Physics Center (DIPC), Paseo Manuel de Lardizabal 4, 20018 Donostia-San Sebastián,Spain

E-mail: maider.ormaza@ipcms.unistra.fr

\begin{abstract}
The manipulation of the molecular spin state by atom doping is an attractive strategy to confer desirable magnetic properties to molecules. Here, we present the

*To whom correspondence should be addressed

${ }^{\dagger}$ IPCMS, CNRS UMR 7504, Université de Strasbourg, 67034 Strasbourg, France

${ }^{\ddagger}$ ICN2 - Institut Catala de Nanociencia i Nanotecnologia, Campus UAB, 08193 Bellaterra (Barcelona),

`Instituto de Física de Rosario, Consejo Nacional de Investigaciones Científicas y Técnicas (CONICET) and Universidad Nacional de Rosario, Av. Pellegrini 250 (2000) Rosario, Argentina

$\S$ Centro de Física de Materiales CFM/MPC (CSIC-UPV/EHU), Paseo Manuel de Lardizabal 5, 20018

"Donostia International Physics Center (DIPC), Paseo Manuel de Lardizabal 4, 20018 Donostia-San
\end{abstract} Spain Donostia-San Sebastián, Spain Sebastián,Spain 
formation of novel magnetic metallocenes by following this approach. In particular two different on-surface procedures to build isolated and layer-integrated Co-ferrocene $(\mathrm{CoF})$ molecules on a metallic substrate via atomic manipulation and atom deposition are shown. The structure as well as the electronic properties of the so-formed molecule are investigated combining scanning tunneling microscopy and spectroscopy with density functional theory calculations. It is found that, unlike single ferrocene, a CoFc molecule possesses a magnetic moment as revealed by the Kondo effect. These results correspond to the first controlled procedure towards the development of tailored metallocene-based nanowires with a desired chemical composition, which are predicted to be promising materials for molecular spintronics.

keywords: ferrocene, spin doping, Kondo effect, tip-assisted manipulation, scanning tunneling microscopy/spectroscopy, density functional theory

The ability to manipulate the magnetic properties and the spin-polarized current through single molecules is at the basis of molecular spintronics. ${ }^{1-4}$ The spin and the electronic structure of the molecule near the Fermi level critically influence the spin-polarization of the current, ${ }^{5,6}$ opening up the tantalizing prospect of tuning the spin polarization through a custom-made chemistry of the molecule and an accurate choice of the electrode material. Within this context, the investigation of single molecules with built-in magnetic moments that are coupled to ferromagnetic, or more generally, to metallic electrodes represents a model playground for exploring novel spintronic concepts. ${ }^{7}$

Many experimental investigations have reported on the possibility of manipulating the molecular spin by metallizing or altering the chemical environment of porphyrin-based ${ }^{8-11}$ or phthalocyanine-based ${ }^{12-18}$ molecules adsorbed on metallic surfaces, or of cobalt complexes coupled to metallic electrodes. ${ }^{19,20}$ Experimental studies of this kind on single metallocenes $\left(\mathrm{MCp}_{2}\right.$ where $\mathrm{Cp}=\mathrm{C}_{5} \mathrm{H}_{5}$ and $\mathrm{M}=\mathrm{Fe}, \mathrm{Co}$, Ni etc.) remain instead limited, despite numerous studies have predicted that metallocene-based nanowires ${ }^{21-29}$ (M-Cp-M-Cp-...) could produce highly spin-polarized charge carriers. The efficiency of this process is influenced by 
the metallic constituents of the nanowires, ${ }^{24,29}$ the wire length, ${ }^{26}$ and by the geometry of the metal-wire contact. ${ }^{25}$ Progress in this area is hampered by the difficulty encountered in synthesizing these molecules in the correct molecular-device environment. The demand for elaborating a prototypical system with atomic-scale control is therefore strong.

We present here a general method for modifying the structure and magnetic moment of a metallocene molecule by coupling it to a single atom. The method is exemplified by using a ferrocene molecule $\left(\mathrm{FeCp}_{2}\right.$, noted $\mathrm{Fc}$ hereafter $)$ and a cobalt atom adsorbed onto a copper surface. Ferrocene represents the most commonly considered metallocene as its anchoring to surfaces has been studied for years ${ }^{30}$ in view of applications ranging from charge storage ${ }^{31}$ to transistors. ${ }^{32}$ By manipulating ferrocene and cobalt with the tip of a low-temperature scanning tunneling microscope (STM) and forcing them to interact with each other, we show that we can produce a small nanowire consisting of Co-Cp-Fe-Cp (noted CoFc hereafter; see Fig. 1e). The upstanding geometry obtained for $\mathrm{CoFc}$ on the surface is ideal for elaborating a single-molecule device, for example by a subsequent connection of the nanowire to a top electrode. To generalize the process to a collection of molecules, we also show that CoFc can be produced through a spontaneous on-surface reaction of cobalt atoms with a molecular layer of ferrocene. Through a combined STM and density functional theory (DFT) study, we then show that unlike $\mathrm{Fc},{ }^{25} \mathrm{CoFc}$ exhibits a magnetic moment. These results are a substantial advance upon existing studies devoted to metallocene nanowires as, to date, the production and magnetic characterization of these systems are restrained to the gasphase. ${ }^{33,34}$ Our findings can be easily generalized to other metallocenes and to ferromagnetic surfaces.

The measurements were performed with a STM working in ultra-high vacuum (UHV) at 4.4 K using a pristine $\mathrm{Cu}(111)$ surface (see experimental details in the Supporting Information). Ferrocene molecules were sublimated in UHV onto the surface held at a temperature below $100 \mathrm{~K}$. A sub-monolayer coverage of Fc molecules gives rise to a molecular decoration of the surface steps, as shown by the 0.2 monolayer coverage in Fig. 1a. According to the 

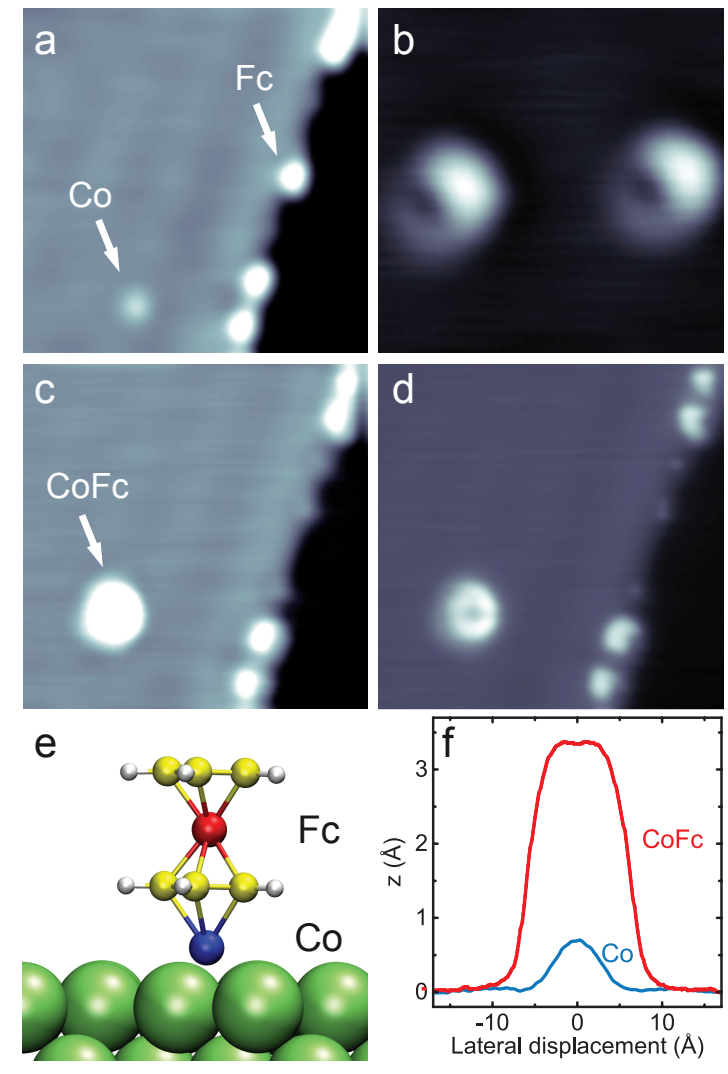

Figure 1: Building process of an isolated CoFc: (a) a Fc molecule at a step edge is picked up with the tip (image acquired with a tunneling bias: $-1 \mathrm{~V}$, current: $20 \mathrm{pA}$; image size: $\left.7 \times 7 \mathrm{~nm}^{2}\right)$, (b) the presence of Fc at the tip apex is asserted by imaging Co atoms $(-50 \mathrm{mV}$, $20 \mathrm{pA}, 2.7 \times 2.7 \mathrm{~nm}^{2}$ ), which then exhibit a molecular pattern, (c) Fc is deposited on top of a Co atom by a tip-atom contact $\left(-1 \mathrm{~V}, 20 \mathrm{pA}, 7 \times 7 \mathrm{~nm}^{2}\right)$. (d) Same image as panel (c) but with a Laplacian filter applied in order to enhance the intramolecular contrast. (e) Chemical structure of $\mathrm{CoFc}$; white, yellow, and red balls represent $\mathrm{H}, \mathrm{C}$, and Fe atoms of the Fc molecule respectively, and green balls represent the $\mathrm{Cu}$ atoms of the $\mathrm{Cu}(111)$ surface. (f) Line profiles of isolated $\mathrm{Co}$ and $\mathrm{CoFc}$ on $\mathrm{Cu}(111)$.

Cp ring-like shape observed in the STM images, ferrocenes are adsorbed individually and vertically at the step edges, $i$. e., with the molecular axis perpendicular to the surface. Subsequent deposition of Co atoms at $4.4 \mathrm{~K}$ leads to a surface in which Co atoms are placed on the terraces and Fc molecules at the step edges (Fig. 1a).

The STM tip is then used as a tool to engineer isolated single CoFc molecules, following the procedure displayed in Figs. 1a-d. The first step towards the formation of a novel CoFc molecule consists in positioning the tip on top of a single Fc molecule and approaching it 
while keeping the bias constant $(-150 \mathrm{mV})$ until an abrupt drop is observed in the current (not shown). This jump in the current indicates that the molecule has been detached from the surface and is now attached to the tip, ${ }^{35}$ as revealed by the after-images shown in Figs. 1bc. In Fig. 1b, a closer view of the Co atom imaged with the Fc-terminated tip is shown. The Co atom, which is round when imaged with a metallic tip as in Fig. 1a, exhibits now a molecular pattern which corresponds to a reverse image of the apex. ${ }^{36-38}$ The ring-shape tip apex corresponds to a $\mathrm{Cp}$ ring of a tilted Fc molecule. ${ }^{39}$ Placing this molecular tip on top of the Co atom and proceeding the same way, $i . e$., decreasing the tip-sample distance until a certain threshold value, we are able to release the molecule from the tip. Figure 1c shows how the targeted Fc molecule has disappeared from the step edge and that a new stable molecule, made up of Co-Cp-Fe-Cp (see sketch in Fig. 1e), has been created in the place where the isolated Co atom was. The new $\mathrm{CoFc}$ molecule presents a ring-shaped feature as shown in Fig. 1d. The corresponding apparent height profiles of an isolated Co atom and a CoFc molecule, $(0.7 \pm 0.1) \AA$ and $(3.4 \pm 0.2) \AA$ respectively, are displayed in Fig. 1f. This molecular building process is fully reproducible.

In order to experimentally confirm that a new molecule is formed, we move the whole CoFc molecule by trapping it using a tip-induced local potential. The STM image of Fig. 2a reveals that when changing the tunneling current from $50 \mathrm{pA}$ to $2 \mathrm{nA}$ (at a constant bias of $-30 \mathrm{mV}$ ) the $\mathrm{CoF}$ follows the tip movement and the image is then acquired by dragging the $\mathrm{CoFc}$ across the $\mathrm{Cu}(111)$ surface. Atomic resolution of $\mathrm{Cu}(111)$ is obtained when $\mathrm{CoFc}$ is dragged, as was evidenced for a Co atom trapped under the STM tip and rastered over the surface. ${ }^{40}$ As expected, a Cu-Cu distance of $2.5 \AA$ is found. Once the current is set back to $50 \mathrm{pA}$ the molecule is released. This supports the idea that CoFc behaves as a unique entity, i. e., the interaction between $\mathrm{Co}$ and $\mathrm{Fc}$ is strong enough to create a new molecular species. Isolated $\mathrm{CoFc}$ is coupled stronger to the $\mathrm{Cu}$ surface compared to $\mathrm{Fc}$, since no isolated $\mathrm{Fc}$ can be found on $\mathrm{Cu}$ terraces.

Indeed, DFT calculations show that the role of the surface is to passivate the otherwise 

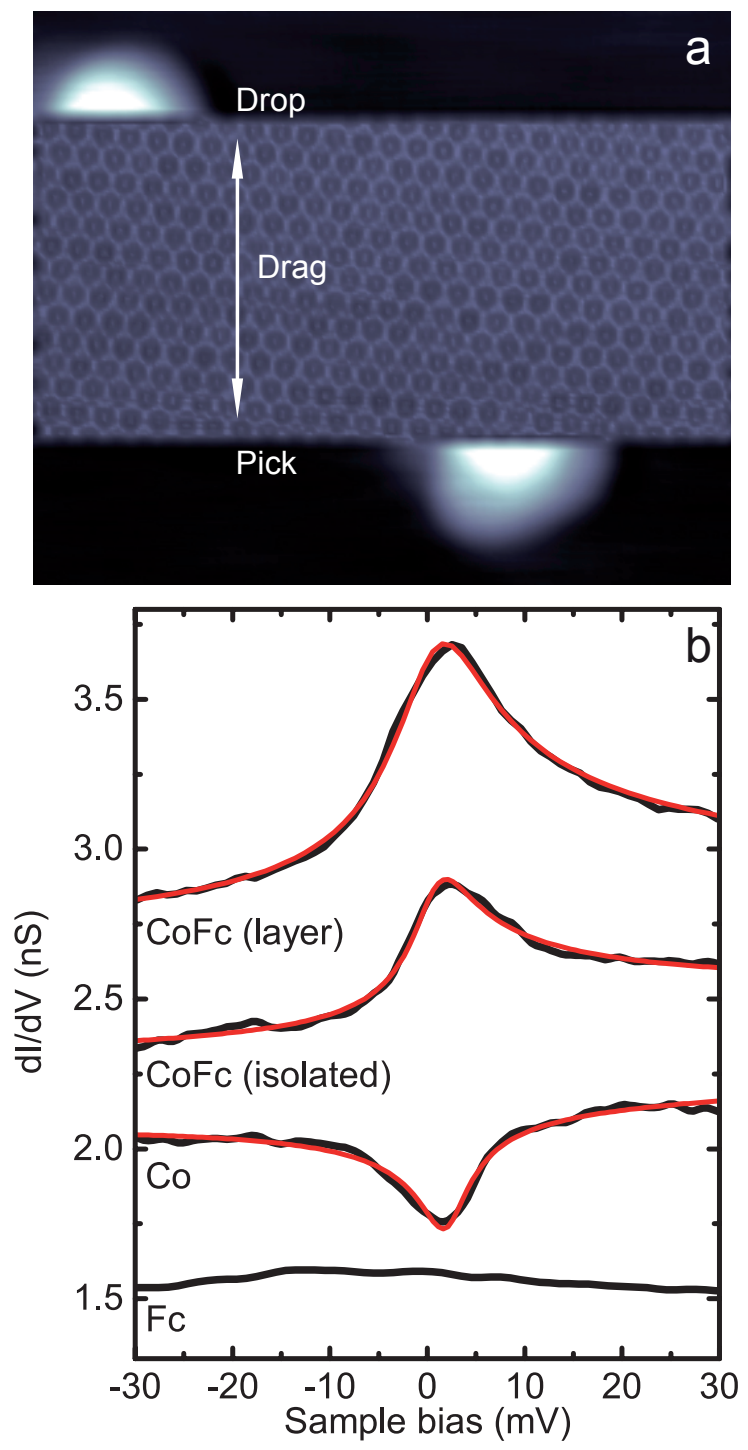

Figure 2: (a) CoFc dragging on $\mathrm{Cu}(111)$ (image size: $6.5 \times 5.2 \mathrm{~nm}^{2}$ ). The dragging parameters are $2 \mathrm{nA}$ and $-30 \mathrm{mV}$. (b) $d I / d V$ spectra including Frota-Fano fits (solid red-line) of isolated $\mathrm{Co}, \mathrm{Fc}, \mathrm{CoFc}$ and layer-integrated CoFc. The curves are shifted by $0 \mathrm{nS}, 0.3 \mathrm{nS}, 0.5 \mathrm{nS}$ and $-6.8 \mathrm{nS}$ respectively. The feedback loop was opened at $-0.03 \mathrm{~V}$ and $0.05 \mathrm{nA}$ for all spectra, except for the the layer-integrated $\mathrm{CoFc}$ were the feedback was opened at $-0.05 \mathrm{~V}$ and $0.5 \mathrm{nA}$. 
reactive $\mathrm{CoFc}$ species. Ab initio calculations have been performed within the DFT as implemented in the VASP code. ${ }^{41,42}$ We have used the generalized gradient approximation for the exchange and correlation functional, ${ }^{43}$ and the DFT $+\mathrm{D} 2$ method proposed by Grimme ${ }^{44}$ to treat the missing van der Waals interactions (more details can be found in the Supporting Information).

These calculations show that due to the location of dangling bonds at the Co edge, the molecule prefers to bind by the Co atom to the surface, although the binding by the $\mathrm{Cp}$ ring of the $\mathrm{Fc}$ unit is also stable. The $\mathrm{Co}-\mathrm{Fc}$ bond can be severed by transferring $1.86 \mathrm{eV}$ to the molecule when it is adsorbed by the $\mathrm{Co}$ atom, and $1.10 \mathrm{eV}$ when adsorbed by the Fc end. Therefore, the surface stabilizes the $\mathrm{CoFc}$, which results to be a molecular unit with a radical character, via passivation of the Co atom. In fact, the molecule is found to be more stable when an extra $\mathrm{Cp}$ is added to form $\mathrm{CpCoFc} .{ }^{45}$ Table 1 summarizes the computed chemisorption properties of a Co atom on an $f c c$ site of the $\mathrm{Cu}(111)$ surface, and their evolution when a Fc molecule is placed on top the Co atom. The Co atom yields more electrons when the Fc molecule is placed on top describing a stronger binding of the Co atom. However, the adsorption geometry of the Co atom is basically unaltered.

The $d I / d V$ spectra near the Fermi energy for isolated Co, Fc and $\mathrm{CoFc}$, recorded with a lock-in amplifier ( $1 \mathrm{mV} \mathrm{rms,} 782 \mathrm{~Hz})$, are shown in Fig. 2b. While no spectroscopic feature is observed for Fc around zero bias, a peak is detected at the Fermi level for CoFc. Its narrow line shape suggests that it corresponds to a Kondo resonance, as evidenced for other organometallic molecules. ${ }^{15,17}$ The Kondo resonances exhibited by Co and $\mathrm{CoFc}$ on $\mathrm{Cu}(111)$ are fitted using a Frota-Fano function (see Supporting Information), ${ }^{46,47}$ as shown in Fig. 2b. The line width of CoFc yields a Kondo temperature of $T_{\mathrm{K}}=(33 \pm 7) \mathrm{K}$, which is higher than that of an isolated Co atom where $T_{\mathrm{K}}=(19 \pm 5) \mathrm{K}$. To confirm the resonance assignment to a Kondo effect, we also compared the spectra of $\mathrm{Co}$ and of $\mathrm{CoFc}$ on the $\mathrm{Cu}(100)$ surface as the Kondo resonance is known to depend on the hybridization of cobalt with the surface. ${ }^{48}$ As expected, ${ }^{49,50}$ on $\mathrm{Cu}(100)$ the Kondo temperature increases yielding, respectively, $(35 \pm 5) \mathrm{K}$ 
for Co and (114 \pm 7$) \mathrm{K}$ for CoFc (see Fig. S1 in the supporting info). The line shape evolution from a resonance to a dip seen in Fig. $2 b$ is quantified by the difference in the Fano fitting parameter found for $\operatorname{CoFc}(q=7 \pm 3)$ compared to $\operatorname{Co}(q=0.10 \pm 0.05)$. This underlines that the orbitals and tunneling paths involved in the Kondo physics are different in the two systems. ${ }^{51}$ The increased value of $q$ for $\mathrm{CoFc}$ is in particular consistent with the higher corrugation of CoFc compared to a Co atom on $\mathrm{Cu}(111)$ which places the tip further away from the surface thereby weakening the direct tunneling into the electron continuum of copper. ${ }^{52}$

The presence of a Kondo effect indicates that CoFc has a magnetic moment. DFT calculations considering the $\mathrm{Cu}(111)$ surface show in particular that the magnetic moment of the Co atom is reduced when bound to Fc due to the increase of occupation of the d-shell. In the molecule, the occupied peaks for the majority density of states (DOS) of Co shift to higher energies compared to an isolated Co, hybridizing with states coming from Fc (Fig. 3). The Fc molecule becomes polarized in spin and the majority spin DOS splits to hybridize with the Co levels. Similar changes occur for the minority spin. The transformation of the DOS due to the $\mathrm{Co}-\mathrm{Fc}$ interaction, reveals the hybridization of the electronic structure forming a molecular unit and the partial depletion of Co levels leading to a reduction of the magnetic moment. In the $\mathrm{CoFc}$ molecule, the computed magnetic moment of the Co atom is in fact $1.33 \mu_{\mathrm{B}}$ (see Table 1), which is much higher than the magnetic moment of Fe in the same molecule $\left(0.12 \mu_{\mathrm{B}}\right)$ or the $\mathrm{Cp}$ rings $\left(0.02 \mu_{\mathrm{B}}\right)$. The magnetic moment of Co on $\mathrm{Cu}(111)$ is in contrast $2.09 \mu_{\mathrm{B}}$, which shows that after the formation of $\mathrm{CoFc}$, the Co atom undergoes a transition from $S \approx 1$ to $S \approx 1 / 2$ revealing the above changes in electronic structure. This corresponds to a charge transfer of $0.43 e^{-}$from the CoFc molecule to the surface, compared to the $0.16 e^{-}$for the isolated Co atom. Therefore, the Kondo effect of CoFc and of isolated Co are different, in agreement with the measured spectral signatures of the Kondo physics. There is a ferromagnetic (FM) coupling between the Fe and Co atoms of CoFc, which is also apparent in the spin density of the molecule, shown in Fig. 3b; the Co-Fe hybridization leads to peaks of the Co atom that coincide in energy with peaks of the Fe atom (Fig. 3a). 


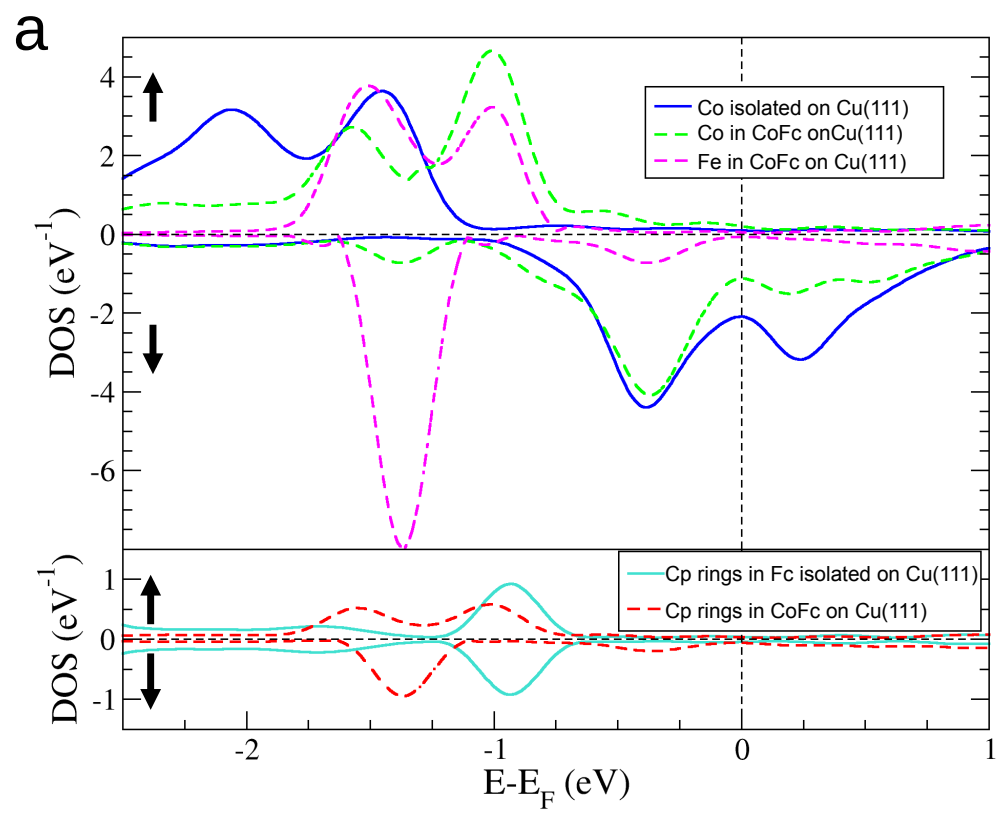

\section{b}

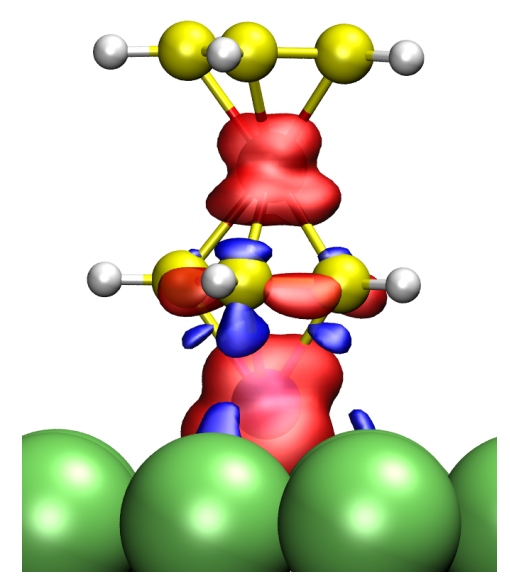

Figure 3: (a) Calculated DOS projected on different atoms of the CoFc molecule (up arrow: majority DOS; down arrow: minority DOS). The dashed lines correspond to the DOS projected onto Co (green), Fe (magenta), and Cp rings (red) when they are part of the new $\mathrm{CoF} c$ molecule. The solid lines show the DOS for Co in the absence of $\mathrm{Fc}$ (i.e. isolated Co) and for Cp rings in the absence of Co (i.e. isolated Fc). (b) Spin density for a CoFc molecule at the Fermi level (majority spin: red; minority spin: blue).

Gas phase calculations of $\mathrm{CoFc}$ show that the FM solution is $98 \mathrm{meV}$ more stable than the antiferromagnetic (broken symmetry) one. The magnetic moment of $\mathrm{CoF} c$ is mainly carried by spin-polarized molecular orbitals (MOs) which are linear combinations of $d_{x z}$ and $d_{y z}$ orbitals of $\mathrm{Co}$ and of $\mathrm{Fe}$, with a contribution from the $p_{z}$-orbitals of the $\mathrm{Cp}$ rings. These MOs, in which the major weight is on the Co atom, are responsible of the observed Kondo effect. Contributions of $\mathrm{Cp}$ rings to this effect are minor due to their negligible magnetic moment.

A different procedure to form the same $\mathrm{CoFc}$ molecule is found when increasing the amount of deposited molecules on the surface and sublimating Co atoms afterwards. A Co coverage above $0.35 \mathrm{ML}$ starts degrading the ferrocene layer, thus, we will limit to a low coverage regime. Figure 4a shows how a molecular coverage up to $0.6 \mathrm{ML}$ gives rise to densely- 

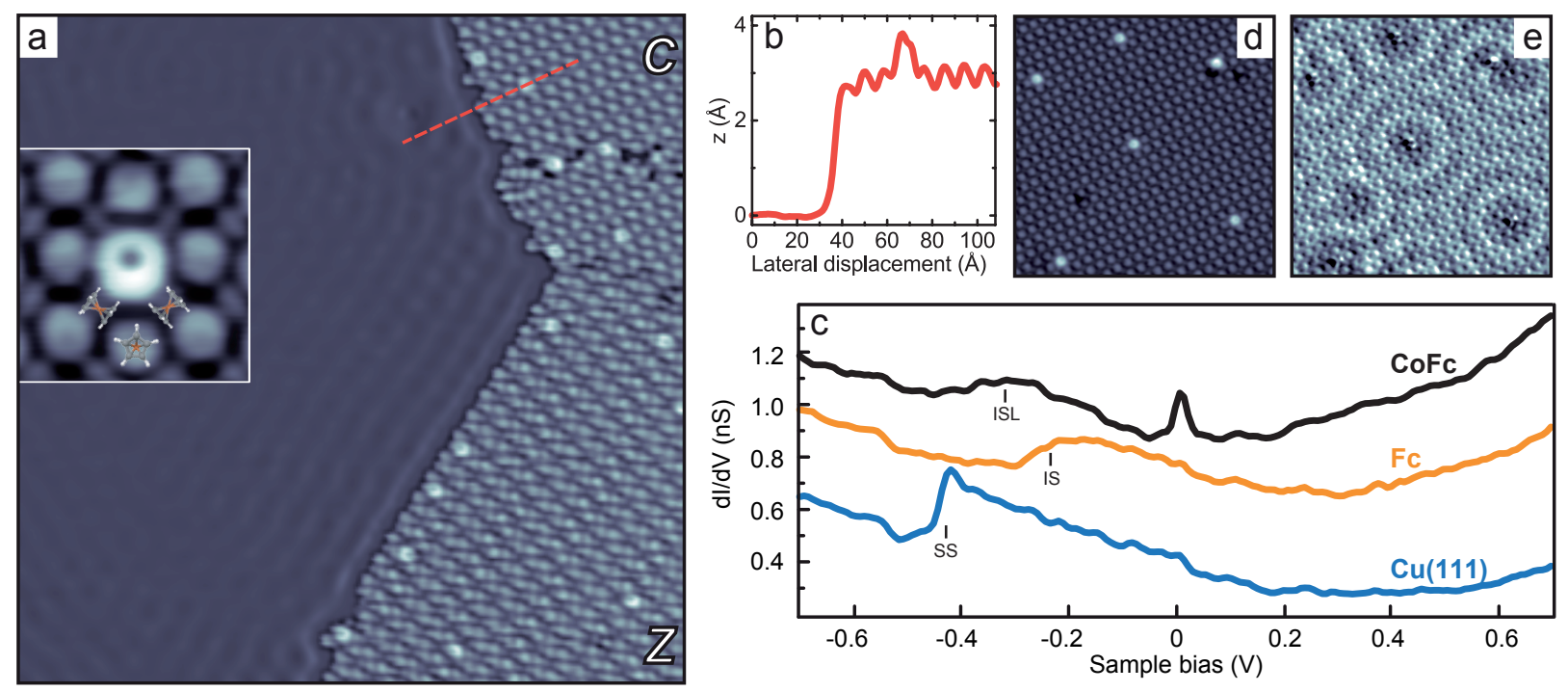

Figure 4: (a) Self-assembly of $\mathrm{Fc}$ on $\mathrm{Cu}(111)\left(-0.05 \mathrm{~V}, 0.5 \mathrm{nA}, 35 \times 35 \mathrm{~nm}^{2}\right)$. The compact and zigzag arrangements are denoted $\mathrm{C}$ and $\mathrm{Z}$, respectively. The bright spots reveal the presence of $\mathrm{Co}$ on the molecular layer. Inset: Close up view of the $\mathrm{CoFc}$ integrated in the compact configuration. A Fc model structure is superimposed on the image to highlight the adsorption geometry of the ferrocene layer. Note that the Co deposition in panel (a) was done at $77 \mathrm{~K}$, as a consequence the Co atoms on copper have diffused to the step edges. (b) Line profile along the red dashed line shown in (a). (c) $d I / d V$ spectra of $\mathrm{Cu}(111), \mathrm{Fc}$ and CoFc. The curves are shifted by $0 \mathrm{nS}, 0.3 \mathrm{nS}$ and $0.5 \mathrm{nS}$ respectively (feedback loop opened at $-1 \mathrm{~V}$ and $0.5 \mathrm{nA}$ ). (d) CoFc image and (e) corresponding constant-current $d I / d V$ map $\left(-0.1 \mathrm{~V}, 0.5 \mathrm{nA}, 20 \times 20 \mathrm{~nm}^{2}\right)$.

packed two dimensional Fc islands. It has been recently shown that Fc molecules physisorb on $\mathrm{Cu}(111)$ following two different possible ways of assembly: compact and zigzag. ${ }^{39}$ Both arrangements, which can be observed in Fig. 4a, consist of a combination of vertical and horizontal molecules, as partially sketched for the compact configuration in the inset. This STM image also shows the surface appearance after exposing it to a small amount of Co atoms (0.05 ML). Among both ferrocene configurations there is no significant difference regarding the adsorption probability and behavior of the Co atoms. Hence, in the following we will only focus on the compact arrangement.

Adsorbed CoFc molecules appear as bright protrusions in the molecular layer with an apparent height of $(0.7 \pm 0.1) \AA$ with respect to vertical Fc molecules and $(3.7 \pm 0.2) \AA$ compared to $\mathrm{Cu}(111)$, as deduced from the line profile along the dashed line displayed in Fig. 4b. 
After a careful inspection of the surface, it can be unambiguously concluded that Co atoms are exclusively adsorbed at the sites of vertically oriented ferrocenes. A closer view into the molecular layer in the inset of Fig. 4a shows that a novel molecule is formed upon Co adsorption, which appears as a ring with a higher intensity in one of the sides. To determine the adsorption of Co in relation with the vertical ferrocene, DFT calculations were performed. From total-energy calculations we conclude that Co atoms prefer to be on an $f c c$ position with respect to the $\mathrm{Cu}$ substrate and below $\mathrm{Fc}$, i.e. in direct contact with the $\mathrm{Cu}(111)$ surface as shown in Figs.5b-c. This conformation is $3 \mathrm{eV}$ more stable than the one with Co on top.

The simulated STM topographic image in Fig.5a, obtained by applying the Tersoff and Hamann theory ${ }^{53,54}$ using the method described by Bocquet et al. ${ }^{55}$ shows how a compact molecular layer would look like if we had several CoFc molecules integrated in the ferrocene layer. The ring-like brightest protrusions correspond to $\mathrm{CoFc}$ and the dimmer ones to Fc. Horizontal molecules are observed as rod-like features between the vertical ones. The striking agreement between simulated and experimental STM images of the CoFc allows assigning the bright ring-like shape in the images to the top $\mathrm{Cp}$ ring of the $\mathrm{CoFc}$ molecule. This ring is moreover tilted approximately by $11^{\circ}$ with respect to the surface normal (Fig.5c) due to the packing of the molecules. ${ }^{39}$ Note that if the Co atom were on top the Fc molecule, according to simulated images (not shown here) no ring-like shape would be observed in the STM images.

To see whether there is any difference between the isolated and the layer-integrated CoFc, the electronic properties of the later one have also been investigated. The $d I / d V$ spectra of $\mathrm{Cu}(111), \mathrm{Fc}$ and $\mathrm{CoFc}$ are displayed in Fig. 4c. The onset of the Shockley surface state (noted SS in Fig. 4c) of $\mathrm{Cu}(111)$ can be easily identified at $-0.44 \mathrm{eV}$; for the physisorbed ferrocene layer, this state is transformed into an interface state (noted IS) with an onset around $-0.26 \mathrm{eV} .{ }^{56}$ In the image and associated $d I / d V$ map of Figs.4d-e the scattering of the interface state of the by $\mathrm{CoFc}$ is shown, revealing a different coupling of $\mathrm{CoFc}$ and 

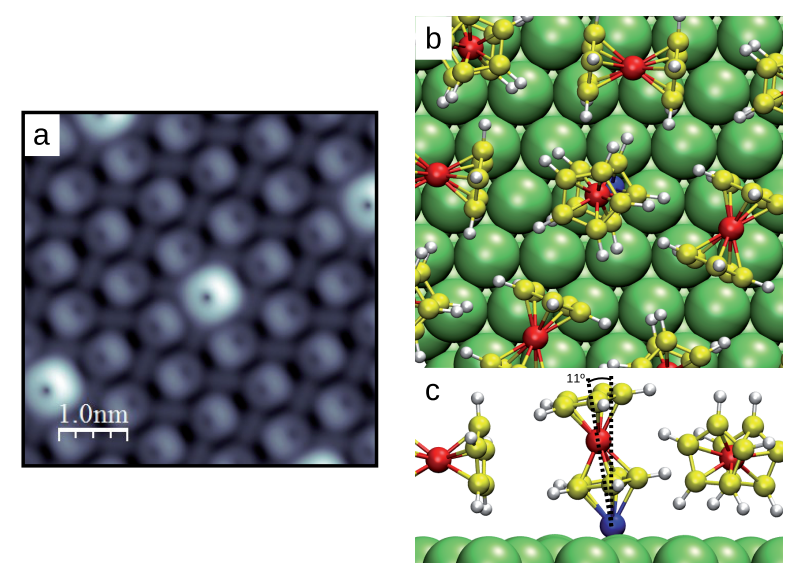

Figure 5: (a) Simulated STM image integrating the local density of states $1 \mathrm{eV}$ above the Fermi energy. The unit cell of the calculation contains a single Co atom per 12 vertically standing Fc molecules. (b-c) Top- and side-view scheme of the compact arrangement with a single Co atom giving rise to a single $\mathrm{CoF} c$ molecule in the unit cell. For clarity only molecules in the middle row of the top view are shown in the side view.

Fc with the substrate. In fact, in the $d I / d V$ spectrum of CoFc a broad resonance is observed around $-0.35 \mathrm{~V}$, which corresponds to a localization of the interface state (noted ISL). ${ }^{52,57-59}$ More noticeable is the narrow peak detected near zero bias, which as in the previous case can be referred to the Kondo effect presented by CoFc. The resonance is again fitted using a Frota-Fano function as shown in Fig. $2 \mathrm{~b}$, from which we obtain $T_{\mathrm{K}}=(38 \pm 6) \mathrm{K}$ and $q=(6 \pm 3)$. Compared to the results obtained for the isolated CoFc we can conclude that both molecules, isolated and layer-integrated, present an experimentally indistinguishable electronic and magnetic behavior.

The computed adsorption properties, Table 1, also show a small variation when going from the isolated molecule to the monolayer. The Co atom stabilizes more the system for the monolayer by a small $5 \%$ change in the binding energy, as a consequence, the Co atom approaches the Fc molecule and separates $0.05 \AA$ from the $\mathrm{Cu}(111)$ surface. However, both charge transfer and magnetic moments are similar for the isolated and the monolayer case, showing the calculated DOS a negligible downshift of the Co peaks when going into the 
monolayer case. Hence, DFT results imply that as in the experiment, both isolated and layer-integrated cases present the same electronic and magnetic behavior. This indicates that the coupling of the molecule with the substrate is similar in both cases and that the neighboring ferrocenes around $\mathrm{CoFc}$ in the layer do not interact strongly, as to modify its properties. This is in agreement with the reported weak ferrocene-ferrocene interaction in the ferrocene monolayer. ${ }^{39}$

Table 1: Adsorption properties of a Co atom, a Co atom bound to a Fc molecule and a Co atom underneath one of the eight Fc molecules for a compact arrangement. The first property is the vertical distance of the Co atom to the topmost layer $\mathrm{d}$ in $\AA$; the second and third ones are the magnetic moment $\mu$ in Bohr magnetons $\left(\mu_{B}\right)$ of Co and Fe atoms, respectively; the fourth property is the change in the number of electrons of the Co atom; and the last one corresponds to the binding energy of Co to the different systems.

\begin{tabular}{c|ccccc}
\hline \hline & $\mathrm{d}[\mathrm{Co}-\mathrm{Cu}](\AA)$ & $\mu[\mathrm{Co}]\left(\mu_{B}\right)$ & $\mu[\mathrm{Fe}]\left(\mu_{B}\right)$ & $\Delta \mathrm{N}[\mathrm{Co}]\left(e^{-}\right)$ & $E_{B}(\mathrm{eV})$ \\
\hline Co & 1.73 & 2.09 & - & -0.16 & -3.32 \\
CoFc (isolated) & 1.70 & 1.33 & 0.12 & -0.34 & -4.04 \\
CoFc (layer) & 1.75 & 1.44 & 0.11 & -0.34 & -4.21 \\
\hline \hline
\end{tabular}

Summarizing, we have demonstrated that it is possible to build new customized ferrocenebased molecules in a controlled way via STM manipulation and that the same molecule is formed spontaneously by atomic doping of the ferrocene layer, highlighting the stability of this molecule. In particular, we have shown that CoFc exhibits a magnetic behavior, as revealed by the observed Kondo resonance. This means that for the first time we have proved that it is possible to change the composition and length of Fc molecules through a in situ chemical reaction. This result can be generalized to other atoms and metallocene molecules, as we have already checked, opening a path to create and study a whole new family of metallocene nanowires coupled to metal electrodes. According to already existing predictions, some of these molecules are half metallic, making them very desirable elements for spintronics. 


\section{Acknowledgement}

We thank B.W. Heinrich and M.V. Rastei for acquiring preliminary data. We also thank M. Vérot, T. Le Bahers and M.-L. Bocquet for fruitful discussions. R.R. and N.L. acknowledge financial support from Spanish MINECO (Grant No. MAT2012-38318-C03-02 with joint financing by FEDER Funds from the European Union). P.A. acknowledges financial support from CONICET. ICN2 acknowledges support from the Severo Ochoa Progam (MINECO, Grant SEV-2013-0295). IPCMS acknowledges financial support from the Agence Nationale de la Recherche through Grant No. ANR-13-BS10-0016, project LabEx NIE and project LabEx CSC. The authors declare no competing financial interest.

\section{Supporting Information Available}

Details of the experimental methods, DFT calculations and Kondo fitting procedure. STM image and STS spectra obtained for $\mathrm{CoF} c$ molecules on $\mathrm{Cu}(100)$.

This material is available free of charge via the Internet at http://pubs.acs.org/.

\section{References}

(1) Xiong, Z. H.; Wu, D.; Vardeny, Z. V.; Shi, J. Nature 2004, 427, 821-824.

(2) Bogani, L.; Wernsdorfer, W. Nat. Mater. 2008, 7, 179-186.

(3) Candini, A.; Klyatskaya, S.; Ruben, M.; Wernsdorfer, W.; Affronte, M. Nano Lett. 2011, 11, 2634-2639.

(4) Urdampilleta, M.; Klyatskaya, S.; Cleuziou, J.-P.; Ruben, M.; Wernsdorfer, W. Nat. Mater. 2011, 10, 502-506.

(5) Rocha, A. R.; Garcia-suarez, V. M.; Bailey, S. W.; Lambert, C. J.; Ferrer, J.; Sanvito, S. Nat. Mater. 2005, 4, 335-339. 
(6) Iacovita, C.; Rastei, M. V.; Heinrich, B. W.; Brumme, T.; Kortus, J.; Limot, L.; Bucher, J. P. Phys. Rev. Lett. 2008, 101, 116602.

(7) Sanvito, S. Chem. Soc. Rev. 2011, 40,3336-3355.

(8) Gottfried, J. M.; Flechtner, K.; Kretschmann, A.; Lukasczyk, T.; Steinrck, H.-P. J. Am. Chem. Soc. 2006, 128, 5644-5645.

(9) Iancu, V.; Deshpande, A.; Hla, S.-W. Phys. Rev. Lett. 2006, 97, 266603.

(10) Tanaka, H.; Ikeda, T.; Takeuchi, M.; Sada, K.; Shinkai, S.; Kawai, T. ACS Nano 2011, 5, 9575-9582.

(11) Auwarter, W.; Ecija, D.; Klappenberger, F.; Barth, J. Nat. Chem. 2015, 7, 105-120.

(12) Zhao, A.; Li, Q.; Chen, L.; Xiang, H.; Wang, W.; Pan, S.; Wang, B.; Xiao, X.; Yang, J.; Hou, J. G.; Zhu, Q. Science 2005, 309, 1542-1544.

(13) Bai, Y.; Buchner, F.; Wendahl, M. T.; Kellner, I.; Bayer, A.; Steinrck, H.-P.; Marbach, H.; Gottfried, J. M. J. Phys. Chem. C 2008, 112, 6087-6092.

(14) Sperl, A.; Kröger, J.; Berndt, R. Angew. Chem. Int. Edit. 2011, 50, 5294-5297.

(15) Komeda, T.; Isshiki, H.; Liu, J.; Zhang, Y.-F.; Lorente, N.; Katoh, K.; Breedlove, B. K.; Yamashita, M. Nat. Commun. 2011, 2, 217.

(16) Lodi Rizzini, A.; Krull, C.; Balashov, T.; Kavich, J. J.; Mugarza, A.; Miedema, P. S.; Thakur, P. K.; Sessi, V.; Klyatskaya, S.; Ruben, M.; Stepanow, S.; Gambardella, P. Phys. Rev. Lett. 2011, 10\%, 177205.

(17) Robles, R.; Lorente, N.; Isshiki, H.; Liu, J.; Katoh, K.; Breedlove, B. K.; Yamashita, M.; Komeda, T. Nano Lett. 2012, 12, 3609-3612.

(18) Krull, C.; Robles, R.; Mugarza, A.; Gambardella, P. Nat Mater 2013, 12, 337. 
(19) Parks, J. J.; Champagne, A. R.; Costi, T. A.; Shum, W. W.; Pasupathy, A. N.; Neuscamman, E.; Flores-Torres, S.; Cornaglia, P. S.; Aligia, A. A.; Balseiro, C. A.; Chan, G. K.-L.; Abrua, H. D.; Ralph, D. C. Science 2010, 328, 1370-1373.

(20) Wagner, S.; Kisslinger, F.; Ballmann, S.; Schramm, F.; Chandrasekar, R.; Bodenstein, T.; Fuhr, O.; Secker, D.; Fink, K.; Ruben, M.; Weber, H. B. Nat. Nanotechnol. 2013, 8, 575-579.

(21) Liu, R.; Ke, S.-H.; Baranger, H. U.; Yang, W. Nano Lett. 2005, 5, 1959-1962.

(22) García-Suárez, V. M.; Ferrer, J.; Lambert, C. J. Phys. Rev. Lett. 2006, 96, 106804.

(23) Liu, R.; Ke, S.-H.; Yang, W.; Baranger, H. U. J. Chem. Phys. 2007, 127, 141104.

(24) Wang, L.; Cai, Z.; Wang, J.; Lu, J.; Luo, G.; Lai, L.; Zhou, J.; Qin, R.; Gao, Z.; Yu, D.; Li, G.; Mei, W. N.; Sanvito, S. Nano Lett. 2008, 8, 3640-3644.

(25) Zhou, L.; Yang, S.-W.; Ng, M.-F.; Sullivan, M. B.; Tan,; Shen, L. J. Am. Chem. Soc. 2008, 130, 4023-4027.

(26) Shen, X.; Yi, Z.; Shen, Z.; Zhao, X.; Wu, J.; Hou, S.; Sanvito, S. Nanotechnology 2009, 20, 385401 .

(27) Yi, Z.; Shen, X.; Sun, L.; Shen, Z.; Hou, S.; Sanvito, S. ACS Nano 2010, 4, 2274-2282.

(28) Yang, J.-F.; Zhou, L.; Han, Q.; Wang, X.-F. J. Phys. Chem. C 2012, 116, 19996-20001.

(29) Morari, C.; Allmaier, H.; Beiuşeanu, F.; Jurcuţ, T.; Chioncel, L. Phys. Rev. B 2012, $85,085413$.

(30) Finklea, H. O. In Electroanalytical Chemistry; Bard, A. J., Rubinstein, I., Eds.; Marcel Dekker, Inc.: New York, 1996; Vol. 19; pp 109-335.

(31) Fabre, B. Acc. Chem. Res. 2010, 43, 1509-1518. 
(32) Itoh, Y.; Kim, B.; Gearba, R. I.; Tremblay, N. J.; Pindak, R.; Matsuo, Y.; Nakamura, E.; Nuckolls, C. Chemistry of Materials 2011, 23, 970-975.

(33) Nagao, S.; Kato, A.; Nakajima, A.; Kaya, K. J. Am. Chem. Soc. 2000, 122, 4221-4222.

(34) Miyajima, K.; Nakajima, A.; Yabushita, S.; Knickelbein, M. B.; Kaya, K. J. Am. Chem. Soc. 2004, 126, 13202-13203, PMID: 15479056.

(35) Eigler, D. M.; Schweizer, E. K. Nature 1990, 344, 524-526.

(36) Schull, G.; Frederiksen, T.; Brandbyge, M.; Berndt, R. Phys. Rev. Lett. 2009, 103 , 206803.

(37) Heinrich, B. W.; Rastei, M. V.; Choi, D.-J.; Frederiksen, T.; Limot, L. Phys. Rev. Lett. 2011, 107, 246801.

(38) Chiutu, C.; Sweetman, A. M.; Lakin, A. J.; Stannard, A.; Jarvis, S.; Kantorovich, L.; Dunn, J. L.; Moriarty, P. Phys. Rev. Lett. 2012, 108, 268302.

(39) Ormaza, M.; Abufager, P.; Bachellier, N.; Robles, R.; Verot, M.; Le Bahers, T.; Bocquet, M.-L.; Lorente, N.; Limot, L. J. Phys. Chem. Lett. 2015, 6, 395.

(40) Stroscio, J. A.; Celotta, R. J. Science 2004, 306, 242-247.

(41) Kresse, G.; Furthmüller, J. Comput. Mater. Sci. 1996, 6, 15.

(42) Kresse, G.; Joubert, D. Phys. Rev. B 1999, 59, 1758.

(43) Perdew, J. P.; Burke, K.; Ernzerhof, M. Phys. Rev. Lett. 1996, 77, 3865.

(44) Grimme, S. J. Comput. Chem. 2006, 27, 1787.

(45) Abufager, P.; Robles, R.; Lorente, N. J. Phys. Chem. C 2015, 119, 12119-12129.

(46) Frota, H. Phys. Rev. B 1992, 45, 1096-1099. 
(47) Pruser, H.; Wenderoth, M.; Dargel, P. E.; Weismann, A.; Peters, R.; Pruschke, T.; Ulbrich, R. G. Nat. Phys. 2011, 7, 203-206.

(48) Choi, D.-J.; Rastei, M. V.; Simon, P.; Limot, L. Phys. Rev. Lett. 2012, 108, 266803.

(49) Knorr, N.; Schneider, M.; Diekhöner, L.; Wahl, P.; Kern, K. Phys. Rev. Lett. 2002, 88, 096804.

(50) Wahl, P.; Diekhöner, L.; Schneider, M. A.; Vitali, L.; Wittich, G.; Kern, K. Phys. Rev. Lett. 2004, 93, 176603.

(51) Huang, P.; Carter, E. A. Nano Letters 2008, 8, 1265-1269, PMID: 18358009.

(52) Madhavan, V.; Chen, W.; Jamneala, T.; Crommie, M.; Wingreen, N. Phys. Rev. B 2001, 64, 165412.

(53) Tersoff, J.; Hamann, D. R. Phys. Rev. Lett. 1983, 50, 1998-2001.

(54) Tersoff, J.; Hamann, D. R. Phys. Rev. B 1985, 31, 805-813.

(55) Bocquet, M.-L.; Lesnard, H.; Monturet, S.; Lorente, N. In Computational Methods in Catalysis and Materials Science; van Santen, R. A., Sautet, P., Eds.; Wiley-VCH: Weinheim, Germany, 2009; pp 199-219.

(56) Heinrich, B. W.; Limot, L.; Rastei, M. V.; Iacovita, C.; Bucher, J. P.; Djimbi, D. M.; Massobrio, C.; Boero, M. Phys. Rev. Lett. 2011, 107, 216801.

(57) Olsson, F.; Persson, M.; Borisov, A.; Gauyacq, J.-P.; Lagoute, J.; Fölsch, S. Phys. Rev. Lett. 2004, 93, 206803.

(58) Limot, L.; Pehlke, E.; Kröger, J.; Berndt, R. Phys. Rev. Lett. 2005, 94, 036805.

(59) Iancu, V.; Braun, K.-F.; Schouteden, K.; Van Haesendonck, C. Phys. Rev. Lett. 2014, 113, 106102. 